\title{
Aprendizaje cooperativo de la creatividad publicitaria a través de anuncios audio y/o visuales*
}

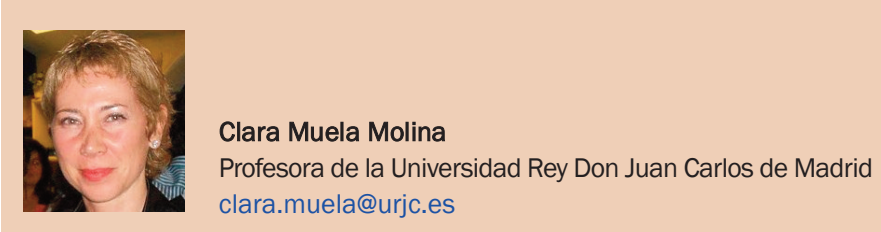

|Fecha presentación: 03/01/2012 | Aceptación: 20/04/2012 |Publicación: 20/06/2012

\begin{abstract}
Resumen
Uno de los principales problemas detectados en la enseñanza de la creatividad publicitaria es el gran desconocimiento que tienen los estudiantes sobre este sector profesional. Así, desde la Universidad Rey Juan Carlos se ha desarrollado un proyecto de innovación docente basado en el aprendizaje cooperativo con el objetivo principal de que los alumnos adquieran un mayor conocimiento de las empresas, profesionales y anuncios más creativos realizados en España, a la vez que ponen en práctica competencias y habilidades que les serán requeridas en su futuro profesional. Para ello, se aplicó la técnica puzzle de Aronson y la prueba del test-retest.
\end{abstract}

Palabras clave: creatividad, publicidad, aprendizaje cooperativo, enseñanza, innovación, universidad, anuncios

\section{Resum}

Un dels principals problemes detectats en l'ensenyament de la creativitat publicitària és el gran desconeixement que tenen els estudiants sobre aquest sector professional. Així, des de la Universitat Rey Juan Carlos s'ha desenvolupat un projecte d'innovació docent basat en l'aprenentatge cooperatiu amb l'objectiu principal que els alumnes adquireixin un major coneixement de les empreses, professionals i anuncis més creatius realitzats a Espanya, al mateix temps que posen en pràctica competències i habilitats que els seran requerides en el seu futur professional. Per a això, es va aplicar la tècnica puzle d'Aronson i la prova del test-retest.

Paraules clau: creativitat, publicitat, aprenentatge cooperatiu, ensenyament, innovació, universitat, anuncis

\section{Abstract}

One of the main problems that has been encountered in teaching of creative advertising is the great lack of knowledge that the students have regarding this professional field. Bearing this in mind, Rey Juan Carlos University has developed a project in innovative teaching based on cooperative learning with an aim for students to acquire a greater knowledge of the companies, professionals and most creative advertisements produced in Spain, while putting into practice competencies and skills that their professional future will require. In order to achieve this, Aronson`s jigsaw technique and the test-retest method were used.

Keywords: creativitat, publicitat, aprenentatge cooperatiu, ensenyament, innovació, universitat, anuncis

* El proyecto titulado “Aprendizaje cooperativo a partir del análisis de recursos audiovisuales: una aplicación de la técnica puzzle al estudio de la creatividad publicitaria" ha sido financiado por la Universidad Rey Juan Carlos en su VI Convocatoria de ayudas a la innovación y mejora de la docencia $2010 / 2011$ y dirigido por la profesora Clara Muela Molina, con la colaboración de los profesores Antonio J. Baladrón Pazos, M ${ }^{\mathrm{a}}$ Ángeles Rubio Gil, M ${ }^{\mathrm{a}}$ Ángeles San Martín Pascal, María F. Sánchez Hernández y de los alumnos ayudantes Patricia Tezanos Bravo y Javier Muñoz García de León. 


\section{Introducción}

La enseñanza de la creatividad publicitaria ha priorizado el estudio de las técnicas creativas, los modelos de resolución de problemas, características psicológicas o intelectuales del sujeto, etapas del proceso o análisis del producto creativo. No obstante, además de las teorías desarrolladas sobre todo desde la psicología, también se ha procurado aplicar esos conceptos al área de la comunicación en general y a la publicitaria en particular; así, la estrategia publicitaria se explica y se practica desde la entrega de un briefing hasta la evaluación de la idea creativa en un ejercicio de role-playing.

Cuando en los antiguos planes de estudio de Publicidad y Relaciones Públicas la asignatura se impartía en los últimos cursos de licenciatura, los docentes detectaban un profundo y grave desconocimiento de los profesionales, las campañas más creativas reconocidas en nuestro país así como en el resto del mundo, y las agencias donde éstos trabajaban; pero esta laguna también se hacía extensible a los creativos que han pasado a la historia por su forma de entender y hacer la creatividad y por sus productos publicitarios. Dichas carencias suponen un gran obstáculo cultural y profesional para el alumno a la hora de enfrentarse al mercado laboral y a la búsqueda de empleo en el ámbito de la publicidad en general y de la creatividad en particular.

El problema principal detectado es que, por lo general, el estudiante de publicidad recibe bastantes contenidos teóricos sobre su especialidad, pero no tiene contacto ni conocimiento sobre la realidad profesional. Aunque esto enraíza en el desinterés y apatía que a veces tiene el alumnado, entendemos que es también responsabilidad del profesorado que ha de desarrollar métodos docentes con los que potenciar ese interés y obligar al alumno a un conocimiento actualizado de la práctica profesional de los estudios que cursa.

En este sentido, uno de los aspectos más interesantes del Plan Bolonia para la adaptación de los grados al Espacio Europeo de Educación Superior (EEES) es la oportunidad que se está ofreciendo a los docentes para probar nuevas técnicas que permitan mejorar el proceso de aprendizaje en los alumnos e incrementar la eficacia docente en la transmisión de conocimientos. Así, la materia de creatividad publicitaria, aunque debe desarrollar una parte teórica sobre los conceptos básicos de esta actividad como parte fundamental de un proceso encaminado a la resolución de problemas para obtener productos originales, también debe eliminar los mitos asociados a la figura del creativo como genio o iluminado. Ésta es, por tanto, una asignatura enfocada a la profesión, pero también un requisito indispensable en cualquier etapa del proceso estratégico de una campaña que, especialmente, se adscribe a un departamento y a unos profesionales que trabajan en grupos creativos.

El proyecto propuesto se justifica, por tanto, por su adecuación para el desarrollo de competencias que el alumno deberá aplicar en el mercado laboral, y por su utilidad para promover en él un mejor conocimiento de la realidad y tendencias actuales de la creatividad publicitaria. En concreto, el libro blanco de los títulos de grado de comunicación describen los contenidos formativos que los estudiantes deben adquirir en esta materia, la mayoría de los cuales se imparten en las clases teóricas magistrales de la asignatura, y otros tantos se estudiarán al año siguiente. En la Universidad Rey Juan Carlos ésta se desdobla en los cursos de $2^{\circ}$ y $3^{\circ}$ de grado con las asignaturas de "Estrategias creativas en publicidad" y "Creatividad en la elaboración del mensaje publici- tario" respectivamente, por lo que tanto la teoría como las prácticas a realizar en $2^{\circ}$ de grado no deben solaparse ni duplicarse respecto a lo que está previsto realizarán los alumnos al año siguiente y que está publicado en el itinerario formativo del grado de Publicidad y Relaciones Públicas. Así, para este proyecto, las competencias generales que han de adquirir los alumnos se refieren al "Conocimiento de los procesos de elaboración de los mensajes publicitarios: métodos, técnicas creativas y los sistemas de análisis de las campañas" y al "Estudio de las estrategias y procesos encaminados a la creación y realización de mensajes publicitarios en los distintos soportes y medios de comunicación" (ANECA 2006: 274).

En cuanto a las competencias específicas, el alumno deberá demostrar, al menos, que intenta desarrollar determinadas destrezas imprescindibles para su futuro profesional, así como esforzarse en adquirir y/o potenciar algunas habilidades que le serán requeridas en el desempeño de su trabajo. No obstante, esto resulta difícilmente evaluable en los escasos seis meses en que se imparte una asignatura ya que son aspectos mejorables a medio o largo plazo, según la personalidad del alumno. Pero sí es función del profesor instarles para que sean conscientes de sus limitaciones pero, sobre todo, para ofrecerles la oportunidad de equivocarse, de cometer errores que les ayudarán a aprender y a descubrir el camino hacia su autoformación. Estas competencias se refieren a tres ámbitos concretos en los que se ha basado el proyecto de innovación: trabajo en equipo, realización y redacción del trabajo y defensa pública del mismo. En concreto serían:

1. "Capacidad para trabajar en equipo desarrollando la apertura personal a través de la comunicación oral y escrita [así como] saber gestionar el tiempo con habilidad para la organización y temporalización de las tareas.”

2. "Capacidad para el análisis objetivo de la realidad, para la síntesis y el juicio crítico. Saber objetivar las tareas y relacionar las causas y los efectos para extraer consideraciones válidas."

3. "Capacidad de comunicar eficazmente con fluidez y riqueza en el contexto profesional (de forma oral y escrita) para el desarrollo y optimización de sinergias grupales y para la toma de decisiones." (ANECA 2006: 296; 337).

Todo este contexto previo determinó el marco y el objetivo general pretendidos para este proyecto. La búsqueda de antecedentes fue estéril, ya que no se encontró nada siquiera aproximado al objeto de estudio que queríamos emprender. No obstante, los trabajos que se podrían aproximar al nuestro por similitudes metodológicas son, por ejemplo, el de Díaz-Lefebvre (2004), que desarrolla un elemento concreto de la técnica puzzle para conseguir alumnos expertos en diferentes áreas, adquiriendo la comprensión y el conocimiento necesarios para explicar los conceptos a sus compañeros en el rol de profesores, y el de Karpova, Marcketti y Barker (2011), con el que se potenciaba algunas dimensiones de la creatividad, como la fluidez, la originalidad o la elaboración. Así, los de mayor afinidad temática son, por un lado, el de Oejo Montano (2004), que transformó su asignatura (Creatividad Publicitaria), en una práctica (realización de una campaña) simulando el trabajo del creativo a partir de un briefing sobre el contenido de la materia. Y, por otra parte, el des- 
arrollo de un festival sobre creatividad publicitaria, donde los propios alumnos eligen a los premiados y organizan todo el evento, repartiéndose las funciones y actuando como si fueran verdaderos profesionales (Perlado y Ramos 2006). Por consiguiente, puede observarse que la mayoría de trabajos relacionados con la enseñanza de la creatividad publicitaria se basan en la técnica del rol-playing.

El proyecto de innovación docente propuesto perseguía como finalidad general que, mediante la aplicación de una metodología didáctica sustentada en el trabajo en equipo y el análisis de recursos audio y/o visuales, los alumnos participantes adquirieran un mayor conocimiento de los productos creativos publicitarios y de los actores implicados en su creatividad, al tiempo que desarrollaban determinadas competencias que les serán exigidas en el mundo laboral. En concreto, el objetivo docente general $\left(\mathrm{O}_{\mathrm{DG}}\right)$ trataba de que los alumnos detectaran y categorizaran las principales tendencias en los procesos y productos publicitarios así como tener conocimiento de las agencias y los profesionales protagonistas que han realizado las campañas más creativas en España, siendo reconocidos como tal en los certámenes de mayor prestigio, enseñando al estudiante las técnicas y dotándole de las herramientas necesarias para el análisis de mensajes audio y/o visuales publicitarios desde la estrategia creativa. Este objetivo general basado en la asunción de conocimientos y derivado del estudio de la creatividad publicitaria nacional, se complementa con otros no mesurables, basados en el desarrollo de competencias específicas para esta asignatura, que luego les serán exigidas en el mercado laboral como:

$\mathrm{O}_{\mathrm{D} 1}$ : capacidad de trabajo en equipo, de asumir responsabilidades individuales y colectivas y de saber negociar y llegar a consensos;

$\mathrm{O}_{\mathrm{D} 2}$ : capacidad de abstracción y síntesis;

$\mathrm{O}_{\mathrm{D} 3}$ : capacidad para una correcta expresión oral y comunicación en público.

\section{Diseño del método y procedimiento}

2.1. El aprendizaje cooperativo aplicado al estudio de la creatividad publicitaria

Para conseguir estos objetivos docentes, se utilizó una metodología de aprendizaje cooperativo (Johnson et al. 1994; Kagan 1994; Slavin 1995) caracterizada porque la acción individual de cada alumno se vincula al resultado del grupo; es decir, los estudiantes trabajan por equipos siendo conscientes de dos cosas: de que la contribución individual de cada uno al grupo es imprescindible para obtener un buen resultado; y de que ninguno puede conseguir un resultado completo y positivo si no es con la ayuda de otros estudiantes. Se refuerza, por tanto, lo que se llama interdependencia positiva, que implica la incompatibilidad entre la competitividad individual y el éxito.

Además de facilitar el aprendizaje e incrementar el conocimiento respecto al objeto de estudio, este método cooperativo mejora el desarrollo personal y social de los estudiantes cuando se respetan unas reglas básicas como, por ejemplo, la de aportar ideas para resolver problemas, escuchar ideas de los otros, que todos tengan la ocasión para hablar, pedir ayuda a todos los miembros del grupo antes de preguntar al profesor o recurrir al consenso para resolver conflictos (Slavin 1995).

De las muchas metodologías de aprendizaje cooperativo se optó por la técnica puzzle de Elliot Aronson ${ }^{1}$ (Aronson et al. 1978) cuyo desarrollo y adaptación al contexto de aplicación, objeto de estudio y objetivo de investigación requería un proceso en tres etapas. En primer lugar, se dividió a los alumnos por grupos de trabajo llamados puzzle, y se asignó a todos los miembros de cada equipo una parte diferente de la tarea hasta el punto de que se convirtiera en un experto en ese ámbito. En segundo lugar, los estudiantes expertos en cada una de las partes de la tarea se juntaron y coordinaron para avanzar en un mejor conocimiento de la parte de la que les tocó ser especialistas. En tercer y último lugar, los expertos regresaron a sus equipos base para transmitir lo aprendido a sus compañeros y componer el puzzle. De este modo, el resultado final es producto no sólo del esfuerzo y responsabilidad recíprocos de los miembros de cada equipo base, sino también de su cooperación con miembros de los otros equipos.

La investigación que a continuación se describe no puede considerarse representativa desde el punto de vista estadístico, pero sí significativa para la consecución de los objetivos docentes fijados al principio del trabajo y para evaluar, por tanto, la eficacia del proyecto de innovación docente. La muestra total está compuesta por los $n=71$ alumnos matriculados en el turno de mañana de la asignatura de Estrategias Creativas en Publicidad impartida en el $2^{\circ}$ curso de grado de Publicidad y Relaciones Públicas de la Universidad Rey Juan Carlos de Madrid. El método aplicado para validar la investigación fue el test-retest, es decir, a los alumnos asistentes a clase se les pasó un cuestionario test al inicio del curso y otro retest exactamente igual al finalizar el proyecto de innovación; el número total de encuestados fue de 58 para el test y 32 para el retest. Este método de investigación permite cotejar la variación de los resultados en dos tiempos diferentes -antes y después del proyecto como es este caso- requiriendo que el tiempo para repetir el cuestionario sea corto, que sea realizado por el mismo investigador en el mismo lugar, bajo las mismas condiciones y utilizando el mismo procedimiento de medición y de procesamiento de datos (Taylor y Kuyatt 1994: 14). Aunque por lo general este método se utiliza en el área de las ciencias exactas como las matemáticas, la física o la medicina, hemos encontrado una investigación (Moro Rodríguez 2007) que lo aplicó a las ciencias sociales, en concreto a la publicidad, tomándolo como referencia y base para aplicarlo a nuestro proyecto.

Aunque consideramos que esta investigación es pionera en cuanto al objeto de estudio y la metodología, la ausencia de estudios previos y de unos antecedentes afines en los que poder basarnos nos hizo asumir unas limitaciones de partida, como la de no haber podido pasar el cuestionario a toda la población, ya que no todos los alumnos asistieron a clase los dos días que se realizó la encuesta, y la de no poder garantizar si son exactamente los mismos sujetos; aspectos que no influyen en el diseño de la investigación, en los resultados ni condicionan los objetivos de la misma. 


\subsection{Desarrollo del proyecto de innovación docente}

La aplicación de la metodología de aprendizaje y el desarrollo del las actividades realizadas siguieron cronológicamente las siguientes etapas:

2.2.1. Evaluación de los conocimientos previos

El primer día de clase se pasó el cuestionario a los alumnos. Este test se componía de 6 tablas referentes a los principales medios publicitarios en España que se correspondían con los subtemas a trabajar. Tres columnas definían los tres ámbitos de estudio: creativos publicitarios, agencias de publicidad destacadas, y campañas conocidas en cada uno de los medios a testar. Y las diez filas permitían el máximo de respuestas de las que no se exigía su exactitud; la petición era que escribieran los nombres de empresas, profesionales y anuncios que conocieran. El enunciado de cada una de las 6 tablas era el siguiente: "Mencione, respecto a la creatividad española en televisión (o en el medio correspondiente), las campañas, creativos y agencias que recuerde hayan destacado en el último año". El fin era evaluar los conocimientos previos que tenían sobre el tema objeto de estudio y poder hacer un análisis comparativo, a través de un retest, respecto a los resultados que se obtuvieran en el cuestionario final, para así concluir al final del proceso sobre la eficacia del proyecto de innovación docente.

2.2.2. Explicación del tema, propuestas de subtemas y constitución de los grupos puzzle

El profesor se encargó de proponer y asignar los subtemas a sus alumnos: la creatividad publicitaria en cine y televisión -audiovisual-, en radio, gráfica - prensa y revistas-, en exterior, en marketing directo o promocional e Internet y móvil. Se tomaron como objeto de análisis los mejores productos publicitarios del año anterior al curso académico, entendiéndose como tales las piezas españolas premiadas en los festivales publicitarios del Club de Creativos -ámbito nacional-, de El Sol -ámbito Iberoamericano- y Cannes ${ }^{2}$-internacional-; todos del año 2010.

En la formación de los grupos, lo importante era garantizar criterios de heterogeneidad, para evitar excesivas diferencias intergrupales y mantener en todos los grupos puzzle niveles similares de motivación y rendimiento académico. Asimismo, era conveniente que los alumnos empezasen a trabajar en equipo con personas que no conocieran o con las que no tuvieran mucho contacto, a modo de simulacro de lo que les sucederá en el ámbito profesional; ya en segundo de carrera, en demasiadas ocasiones, los profesores nos encontramos con grupos de trabajo muy consolidados, endogámicos y poco flexibles a un posible nuevo integrante. Por tanto, para la formación de los grupos se siguió la lista oficial de alumnos matriculados y publicada en el Portal Servicios de la Universidad por orden alfabético.

Para los alumnos expertos, el profesor señaló áreas de trabajo en que los grupos podrían dividirse las tareas para ser más operativo y productivo, siendo éstas las siguientes: coordinador del grupo o responsable del correcto y eficaz funcionamiento del mismo; documentación y estudio de la bibliografía y publicaciones científicas relacionadas con cada subtema; manejo e investigación a través de las revistas especializadas y profesionales u otras fuentes digitales; estudio de las agencias y creativos más destacados; análisis de las campañas más creativas; estudio de caso $^{3}$; y redacción del informe-trabajo final.

2.2.3. Entrega de material y recursos a los alumnos

El material audiovisual se adquirió directamente a través de las asociaciones organizadoras de los certámenes publicitarios de creatividad que editan en DVD, u otro formato comercial accesible, el palmarés de cada año, y se fue pasando a cada grupo para que seleccionaran las campañas que cada cual debía analizar. Finalmente, se facilitó a los grupos puzzle una ficha de análisis para aplicarla a cada anuncio que habían de estudiar. También el profesor iba facilitando otro tipo de material para la realización del trabajo a través del campus virtual como las normas de edición, extensión y formato del mismo, índice de contenidos, esquema de la presentación, ejemplos ilustrativos de casos reales, etc.

2.2.4. Desarrollo del proceso de aprendizaje cooperativo

Cada alumno individualmente desarrolló, en primer lugar, la tarea que le había sido asignada según el subtema del grupo. En segundo lugar, en su calidad de experto tuvo que coordinarse con los otros especialistas de diferentes subtemas para una puesta en común del conocimiento al que habían llegado respecto a problemas encontrados y soluciones propuestas, fuentes, compartir información o facilitar la que no fuera útil para uno pero pudiera aprovecharla otro, etc. Posteriormente volvería a su equipo base para transmitir ese conocimiento al resto de componentes del mismo. Todo este proceso se desarrolló a lo largo del semestre dedicando dos clases a trabajar los grupos por las dificultades que un trabajo de estas características, al que no están acostumbrados, conllevaba. Asimismo, fueron numerosas las tutorías solicitadas y dedicadas a la resolución de problemas así como las planteadas por correo electrónico o a través del campus virtual.

Cada uno de los grupos fue coordinado por un alumno en calidad de representante y portavoz que fue elegido por sus propios compañeros, encargándose de la correcta dinámica del grupo, de la consecución de los objetivos en forma y fecha y de la interlocución con el profesor.

2.2.5. Evaluación de los resultados obtenidos

Una vez realizado el trabajo cooperativo por parte de los alumnos, todos hicieron entrega el mismo día de un informe donde se detallaba todo el proceso realizado, conclusiones, fuentes, etc., enviando, al mismo tiempo, una copia del mismo por correo electrónico. Al final del curso, el profesor creó una carpeta con todos los archivos enviados, que fue adjuntada y remitida a los coordinadores de cada grupo a través del correo electrónico.

2.2.6. Presentación de los trabajos

Las fechas de exposición fueron asignadas de forma aleatoria para que todos los grupos contaran con un tiempo similar de ensayo. También al azar se eligieron las personas

${ }^{2}$ El festival Internacional de Cannes no se pudo facilitar a los alumnos por lo costoso de la copia. No obstante, ellos fueron capaces de encontrar la mayoría de anuncios y campañas premiadas a través de Internet.

${ }^{3}$ El estudio de caso se incluyó como parte del trabajo obligatoria para animar a los alumnos a entrevistarse con creativos publicitarios en sus agencias y preguntarles sobre alguna campaña realizada por ellos. Se trataba de que los estudiantes consiguieran información adicional a la obtenida y profundizar en aspectos sobre los que se habían documentado previamente referentes, por ejemplo, al problema del anunciante y el de comunicación, la estrategia creativa, el público objetivo, el producto, la investigación realizada, el proceso creativo, la generación de ideas, el concepto creativo, etc. Y, aprovechar para preguntarle sobre la profesión del creativo, sus perfiles, requisitos, formación, etc. 
que, en representación de todo el grupo, defendieron cada trabajo en público; esto obligó a todos los miembros a prepararse por igual la presentación y a ensayarla juntos para medir el tiempo de exposición fijado en, exactamente, 20 minutos. Para garantizar la asistencia y atención del resto de compañeros los días de la exposición, se les informó que, al finalizar cada una, el profesor podía coger la lista de matriculados en su asignatura y preguntar a cualquier alumno sobre el trabajo que se acababa de defender, y si la persona citada no estaba en clase o no sabía responder, se le podía penalizar con puntuación negativa.

La evaluación se compuso de dos partes. En primer lugar, la calificación a partir del trabajo entregado por cada grupo y, por otra parte, de su exposición pública en el aula. El objetivo es que la calificación final fuese la misma para todos los integrantes, puesto que el resultado colectivo y no el individual es el resultante de aplicar la metodología de aprendizaje cooperativo; la nota es la del equipo de trabajo porque se valora el progreso del grupo y no la competencia individual con los otros.

2.2.7. Evaluación del proceso

A la semana siguiente de la exposición de todos los trabajos, se hizo el retest (pasar el mismo cuestionario del pri- mer día de curso) para contrastar hasta qué punto se habían sido conseguidos los objetivos del proyecto de innovación docente propuesto.

\section{Resultados}

El proyecto implicó una innovación en el método docente habitualmente utilizado para la enseñanza de la creatividad publicitaria, puesto que supuso pasar de una metodología clásica, en la que el alumno es un sujeto pasivo y el profesor un trasmisor de conocimientos, a otra en la que el estudiante se convierte en parte activa de su proceso de aprendizaje. Asimismo, el proyecto supuso una apuesta por la utilización de recursos audiovisuales desde los cuales propiciar el aprendizaje del estudiante. Precisamente el estudio de los lenguajes audio y/o visuales es básico en la asignatura implicada en el trabajo, aunque dicho proceso de enseñanzaaprendizaje necesita desarrollarse también a partir de recursos textuales: bibliografía, hemerografía, artículos científicos, entrevistas en profundidad, etc.

Las Tablas 1, 2 y 3 evidencian que, con este método, se ha conseguido el $\mathrm{O}_{\mathrm{DG}}$ ya que los datos muestran como ha incrementado el conocimiento de los profesionales, agencias y campañas más destacados por su creatividad en España

\begin{tabular}{|c|c|c|c|c|c|c|c|c|c|c|c|c|}
\hline \multirow{2}{*}{$\begin{array}{c}\text { Número de } \\
\text { Respuestas }\end{array}$} & \multicolumn{9}{|c|}{ Creatividad en Cine y Televisión } & \multicolumn{5}{c|}{ Creatividad en Radio } \\
\hline & Campañas & \multicolumn{2}{|c|}{ Creativos } & \multicolumn{2}{|c|}{ Agencias } & \multicolumn{2}{c|}{ Campañas } & \multicolumn{2}{c|}{ Creativos } & \multicolumn{2}{c|}{ Agencias } \\
\hline & Test & Retest & Test & Retest & Test & Retest & Test & Retest & Test & Retest & Test & Retest \\
\hline $\mathbf{o}$ & 25,9 & 21,9 & 86,3 & 46,9 & 41,4 & 12,5 & 84,5 & 65,6 & 100 & 84,4 & 98,3 & 43,7 \\
\hline $\mathbf{1}$ & 29,3 & 18,7 & 13,7 & 31,3 & 15,5 & 15,6 & 13,8 & 12,5 & & 3,1 & 1,7 & 37,6 \\
\hline $\mathbf{2}$ & 18,9 & 28,1 & & 15,6 & 13,8 & 15,6 & 1,7 & 15,6 & & 9,4 & & 6,2 \\
\hline $\mathbf{3}$ & 12,1 & 9,4 & & 3,1 & 10,30 & 12,5 & & 6,3 & & 3,1 & & 12,5 \\
\hline $\mathbf{4}$ & 6,9 & 6,3 & & & 5,3 & 9,3 & & & & & & \\
\hline $\mathbf{5}$ & 3,3 & 3,1 & & & 5,3 & 15,6 & & & & & & \\
\hline $\mathbf{6}$ & & 9,4 & & 3,1 & 3,3 & 12,5 & & & & & & \\
\hline $\mathbf{7}$ & 1,8 & 3,1 & & & 3,3 & & & & & & & \\
\hline $\mathbf{8}$ & 1,8 & & & & 1,8 & 3,2 & & & & & & \\
\hline $\mathbf{9}$ & & & & & & 3,2 & & & & & & \\
\hline $\mathbf{1 0}$ & & & & & & & & & & & & \\
\hline Total & $100 \%$ & $100 \%$ & $100 \%$ & $100 \%$ & $100 \%$ & $100 \%$ & $100 \%$ & $100 \%$ & $100 \%$ & $100 \%$ & $100 \%$ & $100 \%$ \\
\hline
\end{tabular}

Tabla 1: Resultados del Test-Retest en medios audiovisuales. Frecuencia de las respuestas en \%. Fuente: Elaboración propia.

\begin{tabular}{|c|c|c|c|c|c|c|c|c|c|c|c|c|}
\hline \multirow{2}{*}{$\begin{array}{c}\text { Número de } \\
\text { Respuestas }\end{array}$} & \multicolumn{9}{|c|}{ Creatividad en Prensa y Revistas } & \multicolumn{5}{c|}{ Creatividad en Exterior } \\
\hline & Campañas & \multicolumn{2}{|c|}{ Creativos } & \multicolumn{2}{|c|}{ Agencias } & \multicolumn{2}{c|}{ Campañas } & \multicolumn{2}{c|}{ Creativos } & \multicolumn{2}{c|}{ Agencias } \\
\hline & Test & Retest & Test & Retest & Test & Retest & Test & Retest & Test & Retest & Test & Retest \\
\hline $\mathbf{0}$ & 86,2 & 50 & 100 & 59,4 & 100 & 46,9 & 91,3 & 40,6 & 100 & 81,3 & 98,3 & 65,6 \\
\hline $\mathbf{1}$ & 13,8 & 21,9 & & 34,4 & & 21,9 & 5,3 & 25 & & 15,6 & 1,7 & 25 \\
\hline $\mathbf{2}$ & & 12,5 & & 6,2 & & 21,9 & 1,7 & 25 & & & & 6,3 \\
\hline $\mathbf{3}$ & & 6,2 & & & & 3,1 & 1,7 & 6,3 & & & & \\
\hline $\mathbf{4}$ & & & & & & 3,1 & & & & 3,1 & & \\
\hline $\mathbf{5}$ & & 3,2 & & & & & & 3,1 & & & & 3,1 \\
\hline $\mathbf{6}$ & & 6,2 & & & & 3,1 & & & & & & \\
\hline $\mathbf{7}$ & & & & & & & & & & & & \\
\hline $\mathbf{8}$ & & & & & & & & & & & & \\
\hline $\mathbf{9}$ & & & & & & & & & & & & \\
\hline $\mathbf{1 0}$ & & & & & & & & & & & & \\
\hline Total & $100 \%$ & $100 \%$ & $100 \%$ & $100 \%$ & $100 \%$ & $100 \%$ & $100 \%$ & $100 \%$ & $100 \%$ & $100 \%$ & $100 \%$ & $100 \%$ \\
\hline
\end{tabular}

Tabla 2: Resultados del Test-Retest en medios gráficos. Frecuencia de las respuestas en \%. Fuente: Elaboración propia. 


\begin{tabular}{|c|c|c|c|c|c|c|c|c|c|c|c|c|}
\hline \multirow{2}{*}{$\begin{array}{c}\text { Número de } \\
\text { Respuestas }\end{array}$} & \multicolumn{3}{|c|}{ Creatividad en Márketing Directo y Promocional } & \multicolumn{6}{c|}{ Creatividad en Internet y Móvil } \\
\hline & Campañas & \multicolumn{2}{|c|}{ Creativos } & \multicolumn{2}{|c|}{ Agencias } & \multicolumn{2}{c|}{ Campanas } & \multicolumn{2}{c|}{ Creativos } & \multicolumn{2}{c|}{ Agencias } \\
\hline & Test & Retest & Test & Retest & Test & Retest & Test & Retest & Test & Retest & Test & Retest \\
\hline $\mathbf{0}$ & 84,5 & 53,1 & 100 & 78,2 & 100 & 56,1 & 91,4 & 43,8 & 100 & 71,9 & 98,3 & 56,2 \\
\hline $\mathbf{1}$ & 8,6 & 15,5 & & 15,6 & & 21,8 & 3,5 & 12,5 & & 9,4 & 1,7 & 18,8 \\
\hline $\mathbf{2}$ & 3,5 & 9,4 & & 3,1 & & 6,4 & 1,7 & 18,8 & & 15,6 & & 6,2 \\
\hline $\mathbf{3}$ & 1,7 & 9,4 & & 3,1 & & & 1,7 & 12,5 & & 3,1 & & 6,2 \\
\hline $\mathbf{4}$ & 1,7 & 6,4 & & & & & 1,7 & 3,1 & & & & 6,2 \\
\hline $\mathbf{5}$ & & & & & & 3,1 & & & & & & \\
\hline $\mathbf{6}$ & & & & & & & & & & & & \\
\hline $\mathbf{7}$ & & 3,1 & & & & 3,1 & & 3,1 & & & & \\
\hline $\mathbf{8}$ & & & & & & & & 3,1 & & & & 3,2 \\
\hline $\mathbf{9}$ & & & & & & 3,1 & & & & & & \\
\hline $\mathbf{1 0}$ & & 3,1 & & & & 6,4 & & 3,1 & & & & 3,2 \\
\hline Total & $100 \%$ & $100 \%$ & $100 \%$ & $100 \%$ & $100 \%$ & $100 \%$ & $100 \%$ & $100 \%$ & $100 \%$ & $100 \%$ & $100 \%$ & $100 \%$ \\
\hline
\end{tabular}

Tabla 3: Resultados del Test-Retest en marketing directo e interactivo. Frecuencia de las respuestas en \%. Fuente: Elaboración propia.

en los principales medios de comunicación. En ellas puede observarse las diferencias de resultados entre los tests y retests. Otro de los hallazgos interesantes es la diferencia encontrada entre los diferentes medios, así la televisión es el que más respuestas concentraba en los tests mientras que en el resto, la mayoría no obtenían respuesta. En el mismo sentido, los estudiantes conocían más campañas y agencias que creativos; sin embargo, en los retest, proporcionalmente, se han obtenido mejores resultados en el resto de medios. Y, por último, en los test, predominan las frecuencias mayores en las respuestas más bajas (de o a 3) mientras que en los retests se dispersan más y los porcentajes disminuyen en esos mismos ítems.

De todas las competencias puestas en práctica, la que mejor han asumido es la del trabajo de grupo. De hecho, aunque unos han funcionado mejor que otros, la valoración final, en general, ha sido muy positiva. Un dato importante es que en bastantes casos, por la propia naturaleza del aprendizaje cooperativo y lo que suponía el trabajo en la evaluación de la asignatura, algunos grupos, preocupados por los resultados, han prescindido de alumnos que intentaban eludir su parte del trabajo y aprovecharse de sus compañeros 4 ; en la mayoría de ocasiones, la expulsión del grupo se hizo al principio, pero en alguna que otra fue, incluso, el mismo día de la exposición. Así, también el trabajo cooperativo ha sido eficaz y ha colaborado en la consecución del $\mathrm{O}_{\mathrm{D} 1}$

Otra de las carencias detectadas en los alumnos se refiere al uso de las fuentes documentales. Prácticamente todos los grupos se han limitado a buscar datos y fuentes en Internet; la biblioteca, los libros y revistas son conceptos alejados de su universo de trabajo. Y esas limitaciones se han evidenciado bastante en la solidez de los argumentos, en la descripción y desarrollo del contenido de los trabajos y en la defensa de sus conclusiones, por lo que el $\mathrm{O}_{\mathrm{D} 2}$ no se ha superado en gran medida.

Respecto al $\mathrm{O}_{\mathrm{D} 3}$, las presentaciones en grupo, por lo general se han notado muy trabajadas, originales y creativas, demostrando un gran sentido de la responsabilidad y $\mathrm{mu}$ - chas ganas por hacerlo bien. No obstante, la excelente puesta en escena no eclipsó, en la mayoría de casos, las carencias del alumno para hablar en público, siendo las exposiciones uno de los puntos débiles del estudiante de Publicidad y Relaciones Públicas.

\section{Conclusiones y discusión}

El desarrollo de este proyecto de innovación docente se entiende como una retroalimentación que permite depurar los posibles fallos y mejorar una metodología adaptada a las exigencias de la nueva realidad universitaria. Así, como señala el libro blanco de las titulaciones de grado, el profesor de creatividad publicitaria debe provocar en el alumno un "acercamiento a la realidad profesional y al quehacer comunicativo, desde la reflexión y el estudio entendido como el conjunto de sus diferentes acciones, especialmente publicitarias" (ANECA 2006: 337). En la medida en que se produce ese aprendizaje activo y el alumno es el protagonista del proceso de enseñanza-aprendizaje, se puede optimizar el rendimiento del estudiante, reduciendo las tasas de fracaso y contribuyendo a una mejor preparación de cara a su inserción laboral.

Este proyecto de innovación docente ha demostrado que el trabajo cooperativo a partir de la técnica puzzle ayuda a facilitar el aprendizaje e incrementa el conocimiento de la realidad profesional, de sus agentes y protagonistas respecto a la creatividad publicitaria. También han experimentado con gran eficacia el trabajo en equipo, la asunción de liderazgos, las dificultades y problemas en la interacción con los compañeros y su solución buscando el consenso intergrupal. Sin embardo, el $\mathrm{O}_{\mathrm{D} 2}$ en cuanto a la capacidad de extraer conclusiones válidas basadas en la experiencia y el análisis, no ha conseguido los resultados previstos inicialmente, por lo que para futuras ocasiones habría que contemplar acciones más concretas en este sentido. También cabe destacar que en la presentación y defensa oral de los trabajos, aunque se notaban los ensayos y el esfuerzo realizado incluso en alumnos con verdadero pánico escénico, para posteriores trabajos debería plantearse otros objetivos concretos para

${ }^{4}$ Para ayudar a los alumnos a solucionar sus problemas con los propios compañeros de grupo y orientarles en la forma de enfrentarse a alguno en beneficio del resto, se les facilitó un documento muy útil para que aprendieran a solventar las fricciones y llegar a consensos entre ellos: "Cómo enfrentarse a los jetas y a los mantas" (http://bioinfo.uib.es/ joemiro/TTrGrupo/jtasmantas.pdf). Texto original de Barbara Oakley titulado: "Coping with Hitchhikers and Couch Potatoes on Teams", extraído de: Oakleyet al. (2004: 32-4). 
la comunicación oral; incluso algún proyecto de innovación específico para el aprendizaje y desarrollo de esta competencia, que es requisito indispensable para el profesional de la Publicidad y del Relaciones Públicas.

Asimismo, aprovechando la ocasión y con el trabajo como excusa, muchos de los grupos han conseguido entrevistas con profesionales para el estudio de caso siendo recibidos en la propia agencia lo que ha supuesto una experiencia muy interesante para muchos y, para la gran mayoría, su primera aproximación al mundo profesional. Y aunque, por lo general, no ha resultado una tarea fácil, es un hecho que han valorado también positivamente como muy enriquecedor.

\section{Referencias bibliográficas}

Agencia Nacional de Evaluación de la Calidad y Acreditación (2006). Libro Blanco. Títulos de Grado en Comunicación. ANECA,

http://www.aneca.es/var/media/150336/libroblanco_comunicacion_def.pdf Fecha de consulta, 01.11.2011.

Aronson, Elliot Et al.(1978). The Jigsaw Classroom. Beverly Hills (CA): Sage Publications.

Díaz-Lefebvre, René (2004). Multiple Intelligences, Learning for Understanding, and Creative Assessment: Some Pieces to the Puzzle of Learning. Teachers College Record, 106, pp. 49-57.

Johnson, David W.; Johnson, Roger T.; Johnson Holubec, Edythe(1994). The New Circles of Learning: Cooperation in the Classroom and School. Alexandria (VA): Association for Supervision and Curriculum Development.

Kagan, Spencer(1994). Cooperative learning. San Clemente (CA): Kagan Publications.

Karpova, Elena; Marcketti, Sara B.; Barker, Jessica (2011). The Efficacy of Teaching Creativity: Assessment of Student Creative Thinking Before and After Exercices. Clothing an Textiles Research Journal, 29, pp. 52-66.

Moro Rodríguez, Marta M. (2007). Educación en valores a través de la publicidad en televisión. Comunicar, 28, pp. 183-189. http://dialnet.unirioja.es/servlet/articulo? codigo $=2259917$

Oejo Montano, Eduardo (2004). Publicidad, educación y creatividad en las aulas. Comunicar, 23, pp. 95-99. http://dialnet.unirioja.es/servlet/articulo?codigo=1049891

Oakley, Barbara et al..; Felder, R.M. \& al. (2004). Turning Student Groups into Effective Teams. Journal of Student Centered Learning, 2 (1), pp. 9-34

http://www4.ncsu.edu/unity/lockers/users/f/felder/p ublic/Papers/Oakley-paper\%28JSCL\%29.pdf

Perlado, M.; Ramos, M. (2006). Aplicación del proyecto de innovación docente "Jóvenes Tocados por la Publicidad" en la Universidad Antonio de Nebrija, http://www.ucm.es/info/tropico/EGIo2/PONENCIAS/perlado\%20ramos.pdf Fecha de consulta, 17.04.2010.
Slavin, Robert E. (1995). Cooperative Learning: Theory, Research and Practice. Englewood Cliffs (NJ): Prentice-Hall.

Taylor, BarryN.; Kuyatt, CrissE. (1994). Guidelines for Evaluating and Expressing the Uncertainty of National Institute of Standards and Technology (NIST) Measurement Results,

http://physics.nist.gov/Pubs/guidelines/TN1297/tn12 97s.pdf Fecha de consulta, 25.10.2011. 